\title{
La marginalisation des minifundia. Le cas vitivinicole roumain face aux politiques publiques
}

The minifundia cutting off. The Romanian winegrowing case faced to public policies

Valérie Villemin, Wladimir Andreff et Étienne Montaigne

\section{OpenEdition}

Journals

Édition électronique

URL : http://journals.openedition.org/economierurale/3290

DOI : 10.4000/economierurale.3290

ISSN : 2105-2581

Éditeur

Société Française d'Économie Rurale (SFER)

Édition imprimée

Date de publication : 21 octobre 2011

Pagination : 129-144

ISSN : 0013-0559

Référence électronique

Valérie Villemin, Wladimir Andreff et Étienne Montaigne, « La marginalisation des minifundia. Le cas vitivinicole roumain face aux politiques publiques », Économie rurale [En ligne], 325-326 | septembredécembre 2011, mis en ligne le 17 octobre 2013, consulté le 05 mai 2019. URL : http:// journals.openedition.org/economierurale/3290; DOI : 10.4000/economierurale.3290 


\title{
La marginalisation des minifundia Le cas vitivinicole roumain face aux politiques publiques
}

\author{
Valérie VILLEMIN • Agro-Montpellier, UMR Moisa, Montpellier \\ Wladimir ANDREFF • Université de Paris 1 Panthéon-Sorbonne, Paris \\ Étienne MONTAIGNE • Agro-Montpellier, UMR Moisa, Montpellier
}

$\mathbf{L}^{2}$ restructuration foncière, mise en place en Roumanie dès 1991, a conduit à une bipolarité marquée des structures agricoles. À côté de grosses exploitations commerciales, s'est développé un important pôle de très petites exploitations, dites de subsistance, caractérisées par leur orientation quasi-exclusive vers l'autoconsommation. Malgré leur importance numérique et productive, ces minifundia sont marginalisés par les politiques publiques, en partie du fait de la méconnaissance de leurs mécanismes économiques de fonctionnement. C'est ce que nous montrerons dans la première partie de cet article ${ }^{1}$.

Dans la seconde partie, sur la base d'enquêtes de terrain dans quatre départements et d'une extrapolation au niveau national, nous explorons la nature et les déterminants des comportements d'une catégorie particulière de ces minifundia : ceux qui, parmi leurs activités, pratiquent la vitiviniculture. Ces exploitations principalement orientées vers l'autoconsommation se distinguent par des choix techniques (vignes hybrides versus Vitis vinifera) et selon la localisation régionale (zone viticole traditionnelle ou non).

Nous utilisons une grille théorique d'analyse mobilisant le paradigme Structurescomportements-performances (SCP) et la théorie évolutionniste, tels que combinés par Moati (1995-1999).

1. La première version de cet article a été communiquée le 2 octobre 2009 au comité de préparation du numéro spécial d'Économie rurale.
Les résultats obtenus nous permettent, dans la troisième partie, d'analyser les effets à terme de la marginalisation actuelle de cette catégorie d'acteurs, tant sur la part captive du marché du vin représentée par les familles des minifundistes que du point de vue de leur impact productif, social, territorial ou de leur influence sur le processus de restructuration.

\section{Les minifundia Une catégorie d'exploitations marginalisée}

La réalité des minifundia est tardivement reconnue en Roumanie tandis que sa définition reste peu partagée. Elle apparait dans les plans nationaux de développement rural, élaborés dans le cadre du processus d'intégration à l'Union européenne (Ghib et BerrietSolliec, 2008). Le document de 2008 définit ainsi les exploitations de subsistance comme étant inférieures à $2 \mathrm{UDE}^{2}$, tandis que les exploitations de semi-subsistance sont comprises entre 2 et 8 UDE et les exploitations commerciales sont supérieures à 8 UDE.

Nous adoptons une définition plus fonctionnelle, où les exploitations sont distinguées selon les débouchés de leur production, comme celle retenue par le premier recensement général agricole (RGA) postcommuniste, en 2002, qui distingue, parmi les exploitations familiales, et aux côtés des unités à statut juridique :

2. Une unité de dimension économique (UDE) correspond à 1200 euros de marge brute par an. 
Tableau 1. Typologie des exploitations agricoles et viticoles roumaines

\begin{tabular}{l|c|c|c|c|c|c}
\hline & \multicolumn{3}{|c|}{ Exploitations agricoles } & \multicolumn{3}{c}{ Exploitations viticoles } \\
& Nombre & Surface & $\begin{array}{c}\text { Surface } \\
\text { moyenne }\end{array}$ & Nombre & Surface & $\begin{array}{c}\text { Surface } \\
\text { moyenne }\end{array}$ \\
\hline $\begin{array}{l}\text { Exploitations de } \\
\text { subsistance }\end{array}$ & $76,3 \%$ & $28,7 \%$ & $1,3 \mathrm{ha}$ & $98,9 \%$ & $70,0 \%$ & $0,1 \mathrm{ha}$ \\
\hline $\begin{array}{l}\text { Exploitations de } \\
\text { semi-subsistance }\end{array}$ & $21,1 \%$ & $21,3 \%$ & $3,5 \mathrm{ha}$ & $0,9 \%$ & $2,6 \%$ & $0,5 \mathrm{ha}$ \\
\hline $\begin{array}{l}\text { Exploitations familiales } \\
\text { commerciales }\end{array}$ & $2,1 \%$ & $3,7 \%$ & $6,3 \mathrm{ha}$ & $0,2 \%$ & $1,7 \%$ & $1,8 \mathrm{ha}$ \\
\hline $\begin{array}{l}\text { Sociétés commerciales } \\
\text { privées }\end{array}$ & $0,2 \%$ & $20,6 \%$ & $385,3 \mathrm{ha}$ & - & $23,0 \%$ & $91,9 \mathrm{ha}$ \\
\hline Autres & $0,3 \%$ & $25,6 \%$ & $281,5 \mathrm{ha}$ & - & $2,7 \%$ & - \\
\hline TOTAL & 4484893 & $15707957 \mathrm{ha}$ & $3,5 \mathrm{ha}$ & 1125325 & $177269 \mathrm{ha}$ & $0,16 \mathrm{ha}$ \\
\hline
\end{tabular}

Source : d'après le recensement général agricole de 2002, et, pour le secteur viticole, les extrapolations de Valérie Villemin.

- les exploitations de subsistance, dont la production est orientée « seulement vers l'autoconsommation »;

- les exploitations de semi-subsistance, dont le surplus est écoulé sur le marché ;

- les exploitations commerciales, dont la production est principalement vendue sur le marché.

Le RGA 2002 reste encore aujourd'hui la seule source fiable de données ${ }^{3}$; c'est à elle que nous nous rapportons (tableau 1).

Si avant 1990, l'existence des minifundia était cantonnée à $10 \%$ des surfaces agricoles (lopins de terre des zones collectivisées et exploitations individuelles des zones non collectivisées), leur nombre a considérablement augmenté avec le démantèlement, à partir des années 1990-1991, des coopératives de production (équivalent des kolkhozes soviétiques), tandis que les

3. Le prochain recensement (2010-2011) est en cours, il permettra d'avoir des données plus actualisées, sachant que les enquêtes partielles de 2005 et 2007 laissent entrevoir une diminution de $13 \%$ des exploitations familiales. Cependant, il ne fait plus la distinction entre exploitations de subsistance et de semi-subsistance. En ce qui concerne le secteur viticole, les extrapolations de 2005 et 2007 montrent de nombreuses incohérences.

4. Cette catégorie réunit les « unités coopératives », les « unités de l'administration publique » (dont les stations de recherche et les terres des universités) et la ligne «autres » (tableau 3 du RGA 2002). sociétés agricoles (SA) héritaient du noyau résiduel. En 2002, 29 \% des surfaces étaient occupées par plus de 3,4 millions d'unités, soit les trois-quarts des exploitations roumaines.

Nos études de terrain (Villemin, 1996, 1997) nous permettent de dégager un « profil type » de minifundia. D'une surface généralement inférieure à 2,5 ha $^{5}$ pour 2 UTA en moyenne, elles pratiquent la polycultureélevage (céréales et maïs, volailles, porcs, et éventuellement 1 à 2 vaches et chevaux) et couvrent la plupart des besoins alimentaires de la famille. Dans certaines régions (graphique 2), la viticulture s'ajoute à ce système $^{6}$; le vin qui en est obtenu couvre surtout les besoins sociaux de convivialité. Le(s) salaire(s) et/ou la(les)retraite(s) d'un ou deux membres du ménage, bien que peu élevé(s), en contexte de fort chômage et d'une économie en cours de consolidation, sont cependant essentiels à l'acquisition des intrants agricoles et à l'achat de biens de consommation. La vente ou le troc de

5. Selon nous, la limite de 2 UDE fixée par le plan de développement rural se rapproche davantage, en taille physique des exploitations de 3 ha, ce qui surestime la catégorie des exploitations de subsistance en défaveur des exploitations de semi-subsistance.

6. La culture de raisins concerne environ une exploitation de subsistance sur trois. 
produits ou sous-produits agricoles (par exemple, les veaux mâles) peuvent y être observés. Cependant, leur caractère informel, anecdotique et soumis aux opportunités ponctuelles explique leur classification dans la catégorie des exploitations de subsistance.

En ce qui concerne le secteur viticole, nos calculs nous permettent d'estimer que les minifundia détenaient $70 \%$ du vignoble en 2002 (avec un maximum de 0,25 ha par exploitation), tandis que $3 \%$ des surfaces se trouvaient dans des exploitations de semisubsistance, $2 \%$ dans des exploitations familiales commerciales et $23 \%$ dans des sociétés commerciales privées. La détention de deux-tiers du patrimoine viticole par les minifundia fait de celles-ci une catégorie incontournable pour la compréhension de la dynamique du secteur.

L'adhésion de la Roumanie à l'Union européenne en 2007, sans modifier la logique de fonctionnement des minifundia, fortement autarcique, conduit cependant à leur marginalisation.

\section{Peu d'éléments de politique économique et sociale en leur faveur}

Généralement, pour accompagner les mesures qui visent le bon fonctionnement des marchés des produits agricoles, la politique des structures s'attache à accompagner l'évolution des exploitations agricoles (Bazin, 2007). Or, selon Ghib et BerrietSolliec (op. cit.) et Râmniceanu (2004), celle-ci est quasi-inexistante en Roumanie ; aucun choix en faveur d'un ou de plusieurs modèles d'exploitation n'est en effet formulé explicitement.

Ainsi en est-il des exploitations de subsistance ou minifundia, pour lesquels aucun projet politique ne semble aujourd'hui défini. Les principales aides européennes sont inaccessibles aux minifundia, du fait de seuils d'accès trop élevés. Ainsi, les aides directes du premier pilier sont attribuées aux exploitations d'une surface supérieure à 1 ha (composées de parcelles d'une surface minimale de 0,3 ha). Des montants financiers minimaux sont requis pour la plupart des aides du programme de développement rural ainsi qu'un cofinancement, ce qui défavorise les acteurs n'ayant pas accès aux ressources financières (Von Hirschausen, 2008). La rente viagère est la seule mesure ciblée sur les minifundia (Ghib, 2009) ; elle vise la cession de terres au profit de structures agricoles plus grandes.

En l'absence de politique agricole ou rurale qui se préoccupe des minifundia, nous pourrions nous attendre à ce qu'ils soient intégrés dans une politique sociale spécifique. Ce n'est pas le cas. Les retraites des agriculteurs sont en moyenne quatre fois inférieures à celles des non-agriculteurs (Duma et al., 2005). Malgré l'importance numérique de la population rurale retraitée et des enjeux de la lutte contre la pauvreté (Mathijs et Noev, 2004), l'agriculteur roumain, contrairement à l'agriculteur polonais par exemple, ne bénéficie pas de statut spécifique.

\section{De fortes restrictions post-adhésion}

L'entrée dans l'Union européenne (UE) de la Roumanie en 2007 s'est accompagnée pour les minifundia d'une série de contraintes, dont l'origine est autant externe qu'interne au pays.

La transposition de l'acquis communautaire se traduit par une législation nationale qui touche tout particulièrement les plus petites exploitations : interdiction des foires aux bestiaux, restrictions sur les innombrables marchés locaux (Lhomel, 2007). L'interdiction de circulation des charrettes, sur les routes nationales, outil de travail largement répandu dans les fermes de subsistance, réduit les marges de manœuvre des minifundistes. Le gouvernement de la Roumanie, parmi les deux propositions faites par la Commission européenne, a choisi le seuil d'attribution des aides directes le plus restrictif, et par conséquent, le plus pénalisant pour les plus petites exploitations. 


\section{Quelques éléments d'explication}

La marginalisation politique et législative des minifundia étonne, compte tenu de leur nombre et de leur place dans la production nationale.

- Un premier élément d'explication est à rechercher dans l'inexistence de la représentation des minifundia dans les syndicats et dans les organisations professionnelles. Dans le secteur viticole par exemple, le principal organisme de filière, l'organisation nationale interprofessionnelle viticole, ne regroupe que les grands acteurs commerciaux. D'autre part, les minifundia n'ont pas de relais politique efficace susceptible de canaliser leurs revendications (Roger, 2008).

- Cette problématique a également déjoué la vigilance des analystes et chercheurs, tant nationaux qu'internationaux. Les quelques rares études sectorielles vitivinicoles publiées (Blaha et Borzeda, 1999 ; Noev et Swinnen, 2004 ; Zarnoveanu et Stefan, 2002, et dans une moindre mesure, Noev 2007) occultent plus de $50 \%$ de la production, car celle-ci n'était pas visible sur les marchés, ni dans les statistiques.

Le recensement général de 2002, qui succède à celui de 1948 (soit plus de cinquante ans de flou statistique !), pallie ce manque et supprime les difficultés des études sur échantillonnages, comme celles menées par Rizov et al. (2001) ou Davis et Gaburici (1998).

- L'année 2002 est également l'année de la première mention par l'Union européenne de l'importance des petites exploitations dans les nouveaux États membres (Commission européenne, 2002), qui s'est traduite ensuite par la mesure concernant les exploitations de semi-subsistance, intégrée par la Roumanie en 2006 dans son plan stratégique national. Il s'agit là cependant d'une toute première prise de conscience ; les exploitations de subsistance n'y apparaissent en effet qu'en contrepartie des exploitations de semisubsistance.

\section{Une catégorie marginalisée car méconnue?}

Les exploitations de subsistance ou minifundia constituent donc une catégorie tardivement reconnue et peu prise en compte dans les politiques européenne et roumaine. Les mesures s'y rapportant sont autant de facteurs de marginalisation : obligation de respecter des normes restrictives et exclusion des schémas d'aides financières. La seule alternative, timidement dessinée dans le cadre d'une politique des structures très floue, est celle de la sortie des minifundistes âgés de l'activité agricole, via la mesure de rente viagère. Cette marginalisation des minifundia par défaut d'objectifs, est pour le moins surprenante au regard de leur importance sociale, économique et territoriale.

Nous émettons l'hypothèse que la marginalisation des minifundia repose en grande partie sur la méconnaissance de leur logique intrinsèque de fonctionnement et sur l'absence d'outils d'analyse, dans le contexte spécifique qu'est celui de la transition économique. Nous allons tenter de démontrer qu'une compréhension fine de leurs mécanismes de survie permet de revoir leur adéquation avec les exigences de l'acquis communautaire ainsi que l'originalité de leur contribution possible à la politique agricole commune et nationale. Nous le ferons en nous basant sur une analyse empirique du secteur vinicole roumain et du fonctionnement des minifundia viticoles, élaborée à partir d'une centaine d'enquêtes d'exploitations de ce type, réalisées de 1999 à 2001 dans quatre départements?

7. Si les valeurs économiques (les calculs de marges) ont évolué depuis 2001, les logiques de fonctionnement des minifundia sont restées identiques d'après notre travail de veille micro et macro-économique. Cet aspect, ainsi que le peu de données existantes sur les exploitations de subsistance, rendent cette partie de notre travail particulièrement intéressante et même d'actualité, lorsqu'on sait que la Commission européenne s'interroge actuellement sur leur situation et leur futur $-c f$. le séminaire de Sibiu des 13-15 octobre 2010, http://enrd.ec.europa.eu/en-rd-eventsand-meetings/enrd-seminars/semi-subsistenceseminar/fr/semi-subsistence-seminar_home_fr.cfm. 
RECHERCHES

Valérie VILLEMIN, Wladimir ANDREFF, Étienne MONTAIGNE

Tableau 2. Indicateurs économiques des systèmes modélisés de culture de la vigne

(en milliers de ROL / ha - prix déflatés et rapportés au mois de juin 2001)

\begin{tabular}{l|c|c|c}
\hline Type d'exploitations & $\begin{array}{c}\text { Minifundia } \\
\text { vignes hybrides }\end{array}$ & $\begin{array}{c}\text { Minifundia } \\
\text { vignes VV }\end{array}$ & $\begin{array}{c}\text { Soc. commerciales } \\
\text { privées }\end{array}$ \\
\hline Rendement en raisins & 10 t/ha & 4 t/ha & 7,7 t/ha \\
Prix du raisin (milliers de ROL) & 2,0 & 2,6 & 3,0 \\
(1) Produit brut & 20000 & 10400 & 22500 \\
(2) Consommations intermédiaires & 2425 & 3707 & 12480 \\
(3) Valeur ajoutée brute [(1)-(2)] & 17575 & 6693 & 10020 \\
(4) Main-d'œuvre & 0 & 0 & 9826 \\
(nombre d'heures) & $(255)$ & $(92)$ & $(64)$ \\
(5) Marge brute [(3)-(4)] & 17575 & 6693 & 194 \\
(6) Amortissements & 860 & 8257 & 7988 \\
(7) Marge nette [(5)-(6)] & +16715 & -1564 & -7794 \\
\hline
\end{tabular}

Source : élaboration par Valérie Villemin

\section{Encadré 1. Les modalités de calcul}

Sous une logique d'autoconsommation, ces indicateurs n'ont pas de valeur commerciale. Ils permettent néanmoins une comparaison entre les systèmes de culture, moyennant une méthodologie de calcul adaptée à la logique d'autoconsommation (Labonne, 1985).

S'il existe un marché de raisins nobles (3000 lei/kg pour les raisins de bonne qualité achetés par les sociétés), ce n'est pas le cas des raisins hybrides. Nous avons donc évalué son prix à une valeur inférieure à celle des raisins nobles de médiocre qualité (2 600 lei/kg), soit à : 2000 lei/kg.

La main-d'œuvre mobilisée dans les minifundia est exclusivement familiale. En contexte de chômage élevé, elle est abondante et sa valeur, calculée par le coût d'opportunité, est nulle. La valeur importante de la main-d'œuvre pour les vignes hybrides s'explique par leur forte vigueur. C'est pourquoi les parcelles observées ne dépassent pas 0,1 ha, ce qui rend leur prise en charge familiale possible.

Pour les minifundia, les amortissements concernent le petit matériel (seaux, houe, etc., d'une durée de vie comprise entre 2 et 5 ans, selon les articles), une pompe vermorel (durée de vie de 10 ans), auxquels s'ajoute, spécifiquement pour les vignes Vitis vinifera, les coûts liés au cheval ainsi que les coûts de plantations (ceps et système de soutien).

\section{Les minifundia viticoles Nature et facteurs déterminants des comportements}

Le choix des départements de Salaj, Vrancea, Calarasi et Constanta couvre la diversité géographique de la Roumanie ainsi que la variété des aires géomorphologiques : respectivement, le plateau de Transylvanie, les collines de piémont des Carpates orientales (Moldavie), les plaines du Danube de l'Olténie et le plateau érodé de Dobroudja (graphique 2, les zones numérotées de 1 à 4).

Dans un premier temps, afin de caractériser les comportements des minifundistes, nous mobilisons les données microéconomiques collectées lors de nos enquêtes d'étude des pratiques de culture et d'entretien de la vigne ; deux trajectoires comportementales se différencient. Elles reposent sur le choix technique : vigne hybride (ou hybride producteur direct - HPD -, issu des croisements des vignes américaines et françaises) versus vigne française Vitis vinifera (VV). Dans un second temps, nous rechercherons les facteurs de choix des minifundistes expliquant l'engagement dans l'une ou l'autre des trajectoires.

Nous abordons les comportements des minifundia conformément à la théorie évolutionniste, comme guidés par des routines mais également par des mécanismes de variation et une capacité d'apprentissage. D'autre part, nous utilisons le modèle de 
Tableau 3. Marges nettes des systèmes de culture et transformation des minifundia hybrides et VV (montants exprimés en milliers de ROL pour 1 tonne de raisin, 500 I de vin, 80 I de tsuica - prix déflatés et rapportés au mois de juin 2001)

\begin{tabular}{l|c|c}
\hline & $\begin{array}{c}\text { Minifundia } \\
\text { Vignes hybrides }\end{array}$ & $\begin{array}{c}\text { Minifundia } \\
\text { Vignes VV }\end{array}$ \\
\hline (1) Marge nette viticole (rapportée à 1 tonne de raisins) & +1676 & -391 \\
\hline $\begin{array}{l}\text { (2) Marge nette vinicole } \\
\text { (pour 500 I de vin, produit à partir de 1 t de raisins) }\end{array}$ & -990 & -756 \\
\hline $\begin{array}{l}\text { (3) Marge nette - distillation } \\
\text { (pour 80 I à partir du marc, issu de 500 I de vin) }\end{array}$ & +925 & +925 \\
\hline Marge nette totale [(1)+(2)+(3)] & +1607 & -222 \\
\hline
\end{tabular}

Moati (1995-1999), qui permet une approche comportementale dynamique des acteurs économiques, et précise le panel des facteurs susceptibles d'influer sur leur comportement. Ces facteurs sont regroupés en grandes catégories, inspirées du paradigme S-C-P : les conditions de base, le régime de concurrence et les performances des acteurs situés sur le marché étudié. En outre, notre approche souligne l'importance des conditions initiales de la trajectoire mais aussi des facteurs inertiels ${ }^{8}$.

\section{Rationalité de la culture à l'échelle de l'exploitation}

Le choix technique vignes hybrides versus Vitis vinifera (vigne $V V$ )

L'étude des pratiques culturales des minifundia nous permet de distinguer deux systèmes :

- l'un centré sur la vigne Vitis vinifera (sur

35000 ha selon le RGA de 2002),

- l'autre sur la vigne hybride (89 000 ha).

Tandis que le premier produit des vins fins, résultat d'une sélection pluriséculaire, le second se caractérise par des vins dits «foxés » et par une grande rusticité : résistance aux principales maladies cryptogamiques, forte productivité (rendement élevé

8. Le lecteur intéressé par la grille de Moati, et par son adaptation aux fins de notre étude, en trouvera une description détaillée dans la publication : CiolosVillemin V. Trajectoires de restructuration industrielle en économie de transition : Le cas de l'industrie vitivinicole en Roumanie. Journées européennes des thèses, Montpellier, juin 2003. par cep) et entretien aisé (taille et multiplication des plants accessibles sans savoir spécifique, par bouturage).

Le tableau 2 présente une série d'indicateurs économiques qui permettent de mesurer la viabilité des cultures à court terme (le cycle annuel de production), à moyen terme (l'entretien des cultures par le remplacement des ceps abîmés) et à long terme (le renouvellement de la culture). Ils ont été calculés à partir d'exploitations types, modélisées sur la base de tout notre échantillon d'enquêtes, selon la méthode présentée par Dufumier et Bergeret (2002). À des fins de comparaison, nous présentons également les résultats économiques moyens des sociétés commerciales privées. L'encadré 1 détaille les modalités des calculs.

Pour les minifundia, les amortissements concernent le petit matériel (seaux, houe, etc., d'une durée de vie comprise entre 2 et 5 ans, selon les articles), une pompe vermorel (durée de vie de 10 ans), auxquels s'ajoute, spécifiquement pour les vignes Vitis vinifera, les coûts liés au cheval ainsi que les coûts de plantations (ceps et système de soutien).

À partir des raisins, les minifundia produisent du vin et de l'alcool, de manière artisanale, sur l'exploitation. Nous détaillons, dans le tableau 3, la marge nette dégagée par la production vitivinicole, pour un minifundia (500 l de vin).

Les marges brutes viticoles sont positives. Elles indiquent un comportement rationnel sur le court terme ; chaque année, les ménages ne dépensent pas davantage 
pour produire du raisin qu'ils ne le feraient s'ils l'achetaient. De facto, tout se passe comme s'ils les acquéraient à un niveau de prix moindre que celui du marché : de huit fois pour les raisins hybrides et de 3 fois pour les Vitis vinifera ${ }^{9}$. Les performances des hybrides sont obtenues grâce aux rendements, à la minimisation des traitements phytosanitaires et à la valorisation de la main-d'œuvre familiale disponible sur l'exploitation.

L'entretien à moyen terme des cultures est cependant problématique pour les minifundia à Vitis vinifera. Tandis que les vignes hybrides peuvent être bouturées par les exploitants, l'acquisition d'un nouveau cep de Vitis vinifera, greffé-soudé et certifié, est coûteuse. C'est pourquoi, les parcelles que nous avons enquêtées présentaient en moyenne $30 \%$ de ceps manquants.

Le solde négatif de la marge nette du système de culture Vitis vinifera indique des difficultés pour assurer le renouvellement à l'identique de la culture à long terme. Même s'il est calculé sur 40 années (au lieu des 25-30 années recommandées), le niveau d'amortissement des parcelles de Vitis vinifera est dix fois supérieur à celui des parcelles plantées en hybrides, du fait essentiellement des coûts de la mise en place du vignoble (palissage, achat des plants) à comparer avec la plantation des hybrides à coût nul (tuteur en bois prélevé dans la forêt et boutures racinées produites par les minifundistes).

La vinification sur l'exploitation permet aux minifundia à Vitis vinifera d'atténuer le solde négatif de la marge nette.

L'analyse des comportements des minifundistes au niveau de la parcelle de vigne montre des pratiques culturales adaptées à leur objectif d'autoconsommation. En comparaison avec les modes de culture intensifs des sociétés commerciales privées, et davantage pour les vignes hybrides que les vignes

9. Ce rapport est celui du produit brut par les consommations intermédiaires [(1)/(2)]
Vitis vinifera, on observe : la simplification de l'itinéraire technique, la réduction des intrants et des débours monétaires, la minimisation des frais de renouvellement des plantations et la valorisation de la main d'œuvre familiale, en conformité avec l'approche théorique de l'économie rurale (Amphoux et al., 1973 ; Badoin, 1971 ; Labonne, op. cit. ; Milhau et Montagne, 1968 ; Pouliquen, 2001b). Si la rusticité des hybrides assure une dynamique de reproduction à l'identique des cultures, les minifundia à vignes Vitis vinifera sont engagés dans une trajectoire de décapitalisation - ce que confirment nos observations des parcelles.

L'alternative technique vigne hybride versus vigne Vitis vinifera, aux conséquences très différentes à long terme, pose la question des déterminants du choix de la culture. Si les vignes hybrides répondent à une logique de reproduction à long terme des minifundia, l'existence de minifundia à Vitis vinifera peut apparaître plus surprenante, du fait qu'elles suivent une trajectoire de décapitalisation.

\section{Un choix de trajectoire marqué par des facteurs inertiels}

L’héritage de Vitis vinifera, condition nécessaire mais non suffisante

Les statistiques nationales révèlent l'absence de plantations de vignes Vitis vinifera par les minifundia depuis 1991 ; cette culture a été héritée de l'époque communiste. C'est pourquoi nous émettons l'hypothèse d'une approche patrimoniale à l'origine du choix des minifundistes en faveur de la trajectoire Vitis vinifera. Nous postulons que toutes les exploitations héritant de Vitis vinifera les cultivent, malgré des coûts d'entretien annuels élevés, en vue de limiter la dégradation de leur capital. Même si elles ne peuvent renouveler les plantations dans l'état actuel de leurs revenus, elles anticiperaient la possibilité, dans un futur proche, de pouvoir valoriser pleinement leur capital, soit par la réalisation d'investissements, soit 
par sa vente. Si cette seule explication était suffisante, nous nous attendrions à ce que tous les héritiers de vignes Vitis vinifera les cultivent et que les minifundia n'en ayant pas hérité plantent des hybrides.

Or nos enquêtes réalisées dans le département de Calarasi infirment cette hypothèse car la plupart des héritiers de Vitis vinifera a abandonné plus ou moins rapidement cette culture. La quasi-totalité du patrimoine en Vitis vinifera hérité en 1990 (943 ha) a été réduit à néant quelques années après sa distribution aux minifundistes; en 1995 ne persistaient déjà plus que 51 ha.

À l'échelle communale, nos investigations révèlent des situations variées, selon qu'il a existé ou non :

1. Des ménages souhaitant bénéficier de Vitis vinifera avec la restitution du foncier.

2. Des sociétés agricoles (SA) dans la commune.

3. Si oui, si celles-ci ont souhaité entretenir les vignes.

Ces trois niveaux constituent les différentes branches de l'arbre de décision présenté dans le graphique 1 ci-après. Ainsi, dans les communes Mircea Voda et Ulmu, où aucun acteur n'a désiré bénéficier de Vitis vinifera, ces dernières ont été arrachées massivement en 1992, lors de la restitution du foncier. Dans les communes de Mânastirea, Cuza Voda et Modelu, où des acteurs ont souhaité bénéficier de Vitis vinifera, ces dernières ont disparu dans les trois années ayant suivi la distribution des parcelles.

Notre hypothèse patrimoniale étant infirmée, il nous faut répondre aux questions suivantes : pourquoi certains acteurs n'ont-ils pas souhaité bénéficier du patrimoine existant en Vitis vinifera ? Et quand ils l'ont souhaité, pourquoi l'ont-ils réduit à néant les années suivantes ?

Les explications avancées par les minifundistes héritiers des vignes lors des entretiens concernent la difficulté technique et le coût élevé de l'entretien des cultures. Leur faible niveau de connaissances en matière d'identification et de lutte contre les divers agents pathogènes de la vigne (oïdium, mildiou) ont conduit à la prolifération des foyers de maladies, affectant de manière irréversible les pieds de vigne et, de ce fait, à leur arrachage. Dans les communes ayant refusé d'emblée la culture des Vitis vinifera, des ingénieurs avaient sensibilisé les minifundistes à ces aspects.

Ainsi, si l'héritage des vignes Vitis Vinifera est une condition nécessaire à leur culture par les minifundistes, il n'est pas une condition suffisante. Le cas du département de Calarasi montre que l'engagement dans la trajectoire Vitis vinifera requiert d'autres éléments, comme l'existence de connaissances spécifiques relatives à la

Graphique 1. Arbre décisionnel du maintien de la culture de vignes Vitis vinifera, département de Calarasi

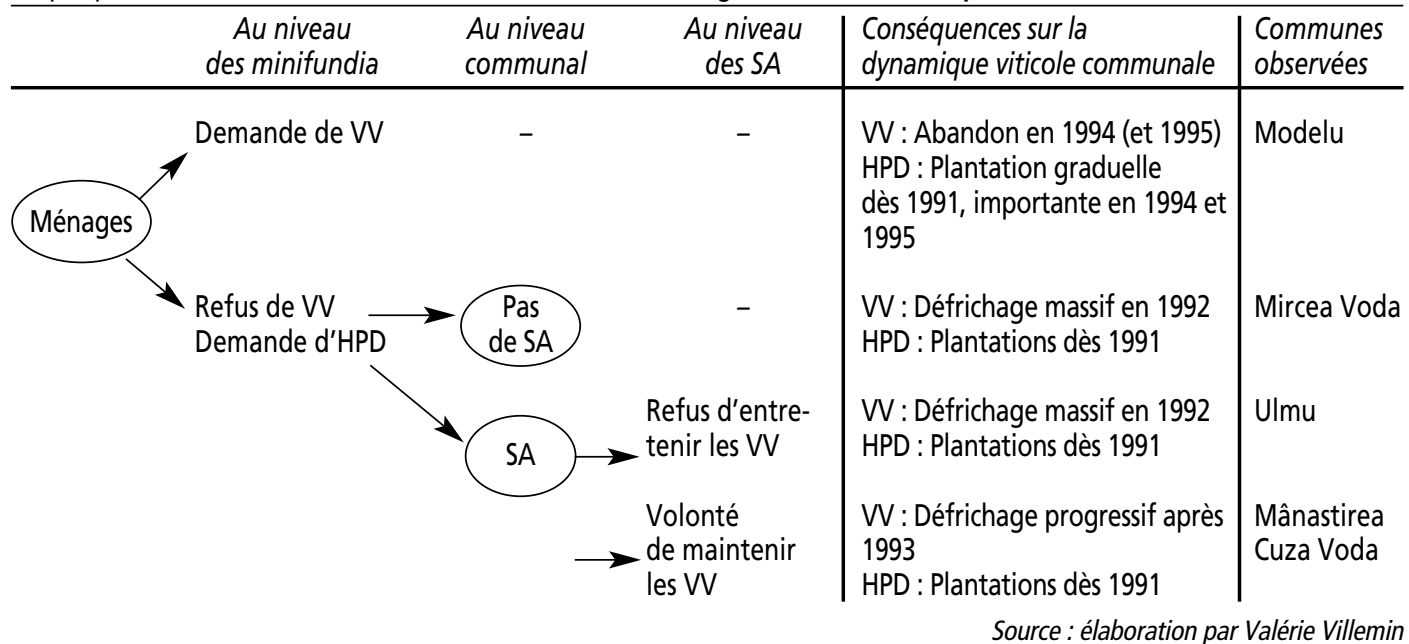


RECHERCHES

Graphique 2. Répartition spatiale des zones selon leur dominante en hybrides producteurs directes (HPD) et Vitis vinifera (VV)

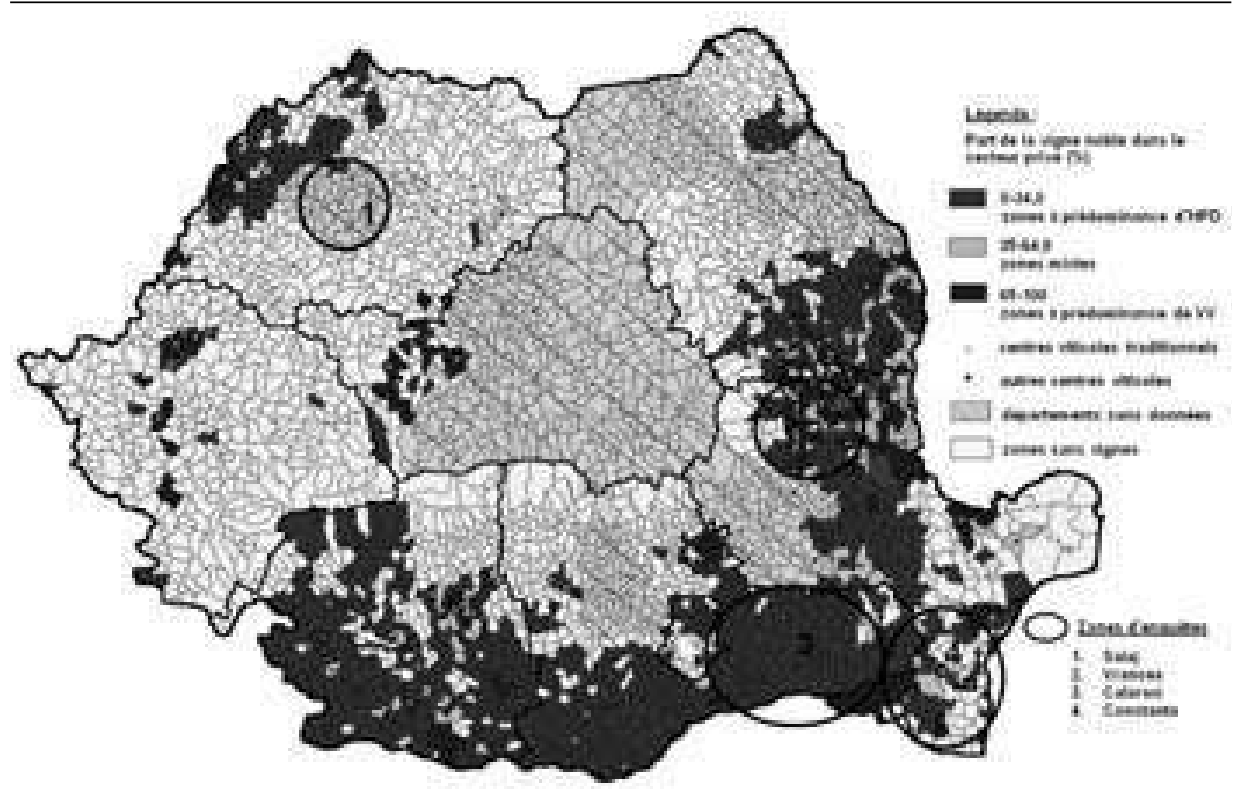

Source : élaboration par Valérie Villemin, à partir des données du projet Relansin ${ }^{10}$ (2001)

culture de la plante. Le rôle des facteurs inertiels, dont l'importance en économie de transition a été mis en évidence par Andreff (1996, 1998, 2003, 2007), apparaît donc ici fondamental. C'est pourquoi, nous proposons de conditionner notre hypothèse patrimoniale à l'existence de facteurs inertiels.

\section{La dimension régionale des trajectoires}

Nous reformulons notre hypothèse ainsi : les exploitations héritant des vignes Vitis vinifera les cultivent, moyennant l'existence, parmi un certain nombre de facteurs favorables, d'un savoir-faire approprié, et ce, malgré des coûts d'entretien annuels élevés, en vue de limiter la dégradation de leur capital. Il devrait donc exister des régions à « savoir-faire » et des régions « sans savoirfaire ». Pour progresser dans notre démonstration, nous assimilons l'existence des savoir-faire viticoles à leur localisation dans des aires de production traditionnelles;

10. Programme national pour la recherche, le développement et l'innovation. nous devrions donc constater que c'est dans ces régions que l'on trouve principalement les minifundia à Vitis vinifera.

En Roumanie, les plantations de vignes Vitis vinifera font l'objet d'autorisations préalables, en conformité avec un zonage des aires favorables. Sous le communisme, les zones de plantation ont été largement étendues, ce qui explique la nouveauté pour certaines régions de la culture des vignes Vitis vinifera et du manque de savoir-faire. Les zones à dominante de Vitis vinifera sont positionnées sur la carte des régions viticoles (graphique 2).

En gris hachuré, sont représentés les départements pour lesquels nous n'avons pas de données et en blanc cassé, les communes sans patrimoine viticole privé. Dans le reste de la carte, trois types de zones sont différenciées. Les zones à dominante d'hybrides (en gris foncé) correspondent aux zones de plaine et sont le plus souvent situées en dehors des régions viticoles traditionnelles. Les zones noires, à dominante de Vitis vinifera, qui se super- 
posent aux centres viticoles traditionnels, à l'exemple de l'arc collinaire subcarpathique de Dealul Mare-Dealul Buzaului-CotestiOdobesti-Panciu, les collines de Moldavie, Murfatlar ou le vignoble de Tarnave (Transylvanie). Enfin, les zones plus claires présentent des zones mixtes; elles sont en nombre très réduit.

Le choix des trajectoires techniques Vitis vinifera versus vignes hybrides s'enracinent ainsi dans une dynamique régionale.

\section{D'autres facteurs inertiels}

À cet argument géographique, nous pouvons enfin ajouter les résultats de nos enquêtes réalisées dans les deux types de région dominantes. Ils permettent de confirmer les facteurs inertiels suivants :

1. la dotation en facteurs (les vignes Vitis vinifera),

2. les routines de production (les savoirfaire), et d'y ajouter les facteurs suivants :

- les routines de consommation (préférences gustatives des minifundistes pour les vins nobles issus de Vitis vinifera versus une appréciation des vins foxés hybrides) et, dans une moindre mesure,

- la disponibilité d'une épargne résiduelle.

Ces héritages du passé constituent les contraintes qui déterminent, dans une perspective évolutionniste, les choix et les trajectoires des exploitations minifundistes.

\section{Deux trajectoires technologiques}

Les comportements des minifundistes suivent une rationalité spécifique. D'une part, nous relevons l'adaptation des pratiques culturales face à un environnement incertain et une chute des ressources monétaires selon un schéma régressif de repli vers l'autoconsommation par la compression des coûts de production, conformément aux approches théoriques de l'économie rurale. D'autre part, les facteurs inertiels, catégorie issue des réflexions théoriques relatives à la transition économique, viennent très clairement expli- quer le choix de culture, hybrides versus Vitis vinifera, opéré par les minifundistes.

Nous observons ainsi deux types de trajectoires, fortement différenciées non seulement d'un point de vue technique et spatial mais aussi par la pérennité effective de leur activité de production vitivinicole.

Les minifundia qui valorisent le caractère rustique des hybrides, suivent une trajectoire d'autoreproduction simple, permise par une absence de prétention qualitative des vins produits. Dans les régions à tradition viticole, les minifundia ayant hérité de Vitis vinifera, mais aussi des routines de production et de consommation associées, cherchent à maintenir leur patrimoine tout en suivant une trajectoire de décapitalisation contenue.

Vingt ans après la redistribution foncière, il est surprenant de constater le maintien d'un patrimoine viticole Vitis vinifera chez des minifundistes, malgré des couts d'entretien et de renouvellement beaucoup plus élevés que pour les vignes hybrides. Seul leur lien avec une certaine tradition explique leur résistance aux pressions économiques ; dans les régions sans tradition, la rationalité purement économique a conduit très vite à leur arrachage.

\section{Une marginalisation vraiment justifiée?}

\section{Les contraintes post-adhésion à l'UE sont-elles bien fondées ?}

En Roumanie, la transition économique s'est accompagnée de la prolifération des cépages viticoles hybrides tandis que la proportion des surfaces plantées en cépages Vitis vinifera, a baissé de $80 \%$ à $50 \%$ entre 1990 et 2007, suscitant très tôt l'inquiétude de l'Union européenne (Commission, 1998). Ces hybrides, issus de la première crise phylloxérique en Europe (Montaigne, 1997) suscitent de fortes craintes pour des raisons :

- de santé publique : les vins qui en sont issus peuvent avoir une teneur élevée en 
méthanol). Six hybrides sont pour cela, interdits par la législation européenne ${ }^{11}$;

- mais surtout, de déstabilisation des marchés : les hybrides sont considérés comme une menace pour la qualité, et ce d'autant plus que les marchés des vins connaissent des déséquilibres chroniques (Montaigne et al, 2005, 2006, 2008).

Suite aux négociations d'adhésion, la Commission européenne a reconnu les variétés hybrides comme partie intégrante du patrimoine viticole roumain bénéficiant de droits de plantation, soit une surface totale de 190000 ha. En outre, les hybrides interdits (30 000 ha) font l'objet d'une dérogation selon laquelle les droits afférant de plantation peuvent être reconnus moyennant leur arrachage jusqu'au 31 décembre $2014^{12}$.

La position de l'UE face aux hybrides laisse de facto à la Roumanie, au nom du principe de subsidiarité, la possibilité de l'arrachage, d'un statu quo (l'arrachage étant facultatif), ou encore d'une restructuration, mais sous financements roumains.

La réponse de la Roumanie est pour le moins ambiguë. D'un côté, elle affiche le désir d'arracher les 30000 ha dans sa stratégie de développement du secteur viticole roumain 2006-2015 ${ }^{13}$, tandis que de l'autre, aucune mesure n'est prévue, ni pour dédommager les exploitants, ni pour les inciter à restructurer leur vignoble avec des cépages Vitis vinifera.

Or les vignes hybrides sont cultivées par des minifundia (environ 90000 ha), sur des surfaces individuelles n'excédant pas 0,1 ha, à des fins d'autoconsommation, conformément à la logique décrite plus haut. La configuration actuelle de la production de vins hybrides en Roumanie ne met donc aucunement en danger les marchés

11. Règlement $n^{\circ} 478 / 2008$, article 24 : Clinton, Herbémont, Isabelle, Jacquez, Noah et Othello. 12. $C f$. le protocole d'adhésion, JO L156 du 21 juin 2005.

13. HG $1^{\circ} 423$ du 17 novembre 2005. européens. Par contre, un arrachage prématuré des hybrides (tant les cépages interdits que les autres hybrides) pourrait avoir selon nous des effets très néfastes pour le marché. Le principal problème auquel se confronte le marché viticole roumain est l'abondance des vins frelatés (boisson frauduleuse produite selon un processus simplifié, moins couteux, peu respectueux des règlements européens et donc biaisant la concurrence) sur le marché domestique : de $30 \%$ à $50 \%$ des vins commercialisés selon les responsables de l'ONIV et du ministère de l'agriculture.

Dans un environnement incertain et pour des ménages à faible pouvoir d'achat, nous soutenons que la production et la consommation de vins issus d'hybrides constitue une barrière à la demande des vins frelatés. Si les minifundistes devaient arracher en masse leurs vignes, ils s'orienteraient vers l'achat de vins peu chers et augmenterait ainsi la demande en vins frelatés de la part de deux millions de consommateurs réguliers.

La connaissance de la logique de fonctionnement des minifundistes nous permet ainsi de conclure que non seulement la culture de variétés hybrides n'affecte pas les marchés mais, au contraire, elle contribue à leur assainissement en contenant la demande en vins frelatés. Nous rejoignons en cela l'analyse de Kostov et Lingard (2002).

La politique viticole actuelle marginalise ainsi les minifundia de manière maladroite, en proposant l'arrachage ou le statu quo. Tandis que la première option - heureusement difficilement applicable en l'absence d'incitations financières -, est dangereuse pour les marchés, la seconde rend coupable les minifundia d'amputer la Roumanie de 30000 ha de patrimoine viticole, et de sa place parmi les grands producteurs. Avec une production moyenne d'environ 5 millions d'hl, sur 2005-2009, la Roumanie occupe la cinquième place en Europe. 


\section{L'accès différencié aux aides exclut les minifundia}

Les exploitations viticoles peuvent bénéficier d'aides directes ainsi que de subventions de reconversion-restructuration du vignoble, mais sous condition de critères de taille. Les aides directes, qui relèvent du premier pilier de la politique agricole commune, sont attribuées aux exploitations d'au minimum 1 ha sur la base de parcelles d'une taille minimale de 0,1 ha (pour la vigne et arbres fruitiers, sinon de $0,3 \mathrm{ha}$ ).

Quant au programme de reconversionrestructuration, il vise à accompagner les producteurs de vin dans leurs efforts d'adaptation à la demande du marché et en faveur d'une meilleure compétitivité. Couvrant jusqu'à $75 \%$ des dépenses éligibles, il répond fort à propos aux besoins de modernisation et de renouvellement d'un vignoble quasidélaissé depuis 1990. Le seuil et le plafond d'accès ont été cependant révisés après les deux premières campagnes de 2007 et 2008 ; les plans de restructuration doivent désormais concerner des surfaces supérieures à 0,5 ha (au lieu de 0,3) et la limite supérieure, initialement fixée à 150 ha a été supprimée. Les parcelles doivent avoir une taille minimale de 0,1 ha, avant et après restructuration.

Compte-tenu de leur taille, les minifundia sont exclues de ces opportunités financières, et donc, privées de la possibilité de modifier leur dynamique de développement vers une trajectoire de recapitalisation pour les minifundistes à vigne Vitis vinifera et une orientation vers la qualité pour les exploitations à vignes hybrides. Nous pouvons nous interroger sur le bienfondé d'une telle marginalisation.

Même s'ils n'interviennent pas sur le marché viticole, les minifundistes le soutiennent de manière substantielle, car ils constituent un marché captif de consommateurs de vins. Les minifundistes cultivant des hybrides pourraient s'orienter à l'avenir, lorsque leur niveau de vie augmentera, vers la consommation, voire la production, de vins de meilleure qualité.

N'est-il pas dommageable pour la filière de faire disparaitre toute une culture populaire du vin avec l'arrachage des hybrides et avec l'extinction des minifundistes Vitis vinifera, au bout - et à bout - de leur trajectoire de décapitalisation, après avoir résisté tant d'années depuis 1991 ? Nous soutenons que l'appui aux minifundia est un élément clef d'une politique de maintien et de développement de la culture du vin et de la qualité, surtout pour un marché domestique inondé de vins frelatés.

\section{Une exclusion administrative}

La principale justification de l'exclusion des très petites exploitations des programmes de financement n'est pas politique mais administrative. La charge est en effet bien lourde pour la toute jeune agence de paiement, passée depuis 2004, et en moins de deux ans, de 16 à 5200 personnes, devant traiter plus de 1,2 millions de dossiers et rassurer Bruxelles au quotidien de la bonne gestion des aides. Cette réalité est à l'origine du relèvement des seuils d'accès aux aides, excluant ainsi quelques centaines de milliers d'exploitations.

Si les contraintes sont fortes et réelles, des raisons administratives peuvent-elles raisonnablement justifier l'exclusion de plus de $70 \%$ d'un vignoble et faire l'impasse sur toute réflexion politique ? Nous pensons que des solutions de nature organisationnelle pourraient être recherchées, afin d'accroître le nombre de bénéficiaires sans pour autant augmenter la charge administrative ${ }^{14}$, avec l'appui de services de conseil spécialisés ${ }^{15}$.

14. Par exemple, pourrait être envisagé le regroupement des demandes d'aides via des entités intermédiaires : mairie, associations agricoles locales ou encore l'association ad hoc des bénéficiaires.

15. Les petites exploitations constituent un public présentant aujourd'hui peu d'intérêt, tant pour la consultance privée que d'État, au regard des faibles sommes mobilisées et de la méconnaissance des mécanismes à l'œuvre au sein de ces organisations. Une structure contrôlée par les agriculteurs, à l'exemple des Chambres d'agriculture françaises, aurait les incitations nécessaires pour assurer une animation efficace. 
En conclusion, les minifundia sont exclus des schémas de financement nationaux et européens, pour des raisons purement administratives. Ils sont ainsi privés d'une possibilité de restructuration, solution alternative au statu quo et à l'arrachage, alors qu'ils entretiennent des liens organiques avec l'ensemble du secteur viticole, y compris avec les marchés - via le marché captif des consommateurs de vins.

La forte charge administrative liée à la mise en œuvre de l'acquis communautaire empêche l'émergence d'une vision politique, qui pourrait considérer les minifundia et les petites exploitations agricoles comme un potentiel et une ressource et non pas comme un fardeau.

\section{L'importance multifonctionnelle}

Si le rôle économique actif des minifundia et l'originalité de leur fonctionnement sont peu connus, il existe un consensus sur leur importance sociale en tant que « tampon », absorbant les surplus de main-d'œuvre libérés par les vagues successives de la restructuration post-socialiste (Kostov et Lingard, 2002, 2004 ; Pouliquen, 2001b).

Dans les montagnes et les pourtours extérieurs des Carpates où les petites exploitations dominent sur plus de $60 \%$ des surfaces, c'est la dimension régionale des minifundia et des petites exploitations que Von Hirschhausen et Guest (op. cit.) mettent en évidence.

Sous l'angle des nouvelles préoccupations qui pèsent de plus en plus sur les réformes de la politique agricole commune, les minifundia présentent paradoxalement une certaine modernité (Darrot et Mouchet, 2005). Ils sont en effet respectueux de l'environnement : les intrants chimiques y sont peu ou pas utilisés, les productions peu intensives et la traction animale y sont de rigueur. Si le faible niveau de mécanisa- tion se traduit par une faible productivité du travail et de la terre (Pouliquen, 2001a), c'est une erreur de tenir les minifundia pour responsables des mauvaises performances nationales agricoles. Selon l'étude de Pouliquen menée pour la Commission européenne (op. cit), les plus gros déficits de productivité se trouvent plutôt dans l'industrie alimentaire et dans les grandes exploitations.

Alors que les débats sur la crise alimentaire sont redevenus d'actualité, les minifundia sont également garants d'une certaine stabilité économique (Kostov et Lingard, 2004), du fait de leur relative autonomie face aux circuits marchands.

Les circuits-courts et la proximité producteurs-consommateurs constituent un facteur de confiance, tant à l'Ouest d'une Europe agricole stigmatisée par la crise de la vache folle qu'à l'Est, marqué par 50 ans de communisme et de double langage.

Enfin, les minifundia jouent un rôle essentiel dans la fixation des populations en zones rurales. L'existence de campagnes vivantes constitue une véritable richesse que beaucoup de pays d'Europe de l'Ouest et même de l'Est - comme la Bulgarie - ont perdu (Von Hirschhausen et Guest, op. cit.) De par son caractère intensif en main-d'œuvre, la vigne valorise bien cette ressource abondante.

Notre propos ici n'est pas de promouvoir les minifundia comme modèle agricole, rural ou social universel. Cependant, plutôt que de marginaliser une frange majoritaire des structures d'un pays, d'ailleurs davantage par maladresse que sur la base d'une réflexion politique, nous soutenons qu'une réflexion fructueuse gagnerait à être engagée en vue de valoriser cette ressource dans le cadre d'une ou plusieurs politiques : sociale, agricole, rurale, régionale, environnementale ou de sécurité alimentaire. 


\section{Conclusions}

La transition post-socialiste a conduit en Roumanie à l'émergence d'un grand nombre de très petites exploitations dont la production est orientée quasi exclusivement vers l'autoconsommation.

Nous nous sommes proposés d'étudier un type particulier de minifundia : ceux qui produisent, entre autres produits, du vin pour la consommation familiale. Bien qu'occupant $70 \%$ du vignoble roumain et regroupant plus de $99 \%$ des unités viticoles, ces exploitations sont les grandes oubliées, tant des économistes que des politiques nationales et européennes. Nous soutenons que cet oubli a un impact négatif sur le secteur vitivinicole roumain.

Notre étude montre une logique originale de fonctionnement des minifundia viticoles, qui repose sur le choix technique vignes hybrides versus vignes Vitis vinifera. Celle-ci intègre tant les contraintes de l'économie agricole que les facteurs inertiels de la transition économique. En région non viticole, on observe ainsi la culture des vignes hybrides, dont la rusticité assure la reproduction simple de l'exploitation. En région viticole, malgré des contraintes économiques fortes, les minifundistes tentent de maintenir la culture de la vigne Vitis vinifera, en suivant une trajectoire de décapitalisation contenue.

L'exclusion des minifundia viticoles des schémas de financements (programme de restructuration et aides du premier pilier) les cantonne à leur trajectoire préadhésion, dans des logiques de consommation de vin médiocre et, pour les minifundia Vitis vinifera, de sortie progressive du secteur. Ce faisant, la filière vitivinicole roumaine s'ampute de précieux alliés porteurs de la culture du vin. Au-delà de leur fonction de production, il ne faut pas oublier que les minifundia sont aussi des consommateurs de vin.

Ainsi, la disparition silencieuse des minifundia Vitis vinifera mais aussi l'arrachage des 30000 ha de variétés hybrides interdites, s'il était décidé, sont des facteurs de déstabilisation du marché domestique roumain. La perte de l'accès au vin autoproduit se traduit en effet par la consommation de boissons concurrentes au vin ainsi qu'à l'augmentation du volume de vins frelatés peu chers, sur un marché déjà bien étendu.

Nous pensons que les minifundia mais aussi les autres exploitations familiales constituent un levier puissant pour la promotion d'une politique de qualité à moyen-terme.

Selon nous, ni l'imprécision des statistiques, ni la complexité de l'analyse requise, ni la lourdeur des traitements administratifs, ne peuvent justifier la marginalisation des minifundia, ni occulter leur importance territoriale, sociale et productive.

Enfin, dans le contexte roumain de bipolarité des structures agricoles, l'exclusion des minifundia élimine la possibilité d'une restructuration via la concentration des terres pour ne prendre en compte que les exploitations commerciales. 


\section{RÉFÉRENCES BIBLIOGRAPHIQUES}

Amphoux B., Cibenel C., Lacombe P., Lifran R. (1973). Coûts de production et concurrence. Le cas de la viticulture méridionale. Économie méridionale, p. 1-12.

Andreff W. (1996). Vers une théorie alternative de la transition post-socialiste : facteurs inertiels, effet de sentier, évolutionnisme. In «L'évolutionnisme: fondements, perspectives et réalisations ». Paris-Sorbonne - METIS, 27 p.

Andreff W. (1998). Les aspects inertiels de la transition. Les cahiers de recherche de l'ESSCA, $\mathrm{n}^{\circ}$ 5, p. 11-90.

Andreff W. (2003). La mutation des économies postsocialistes. Une analyse économique alternative. Paris, L'Harmattan, $368 \mathrm{p}$.

Andreff W. (2007). Économie de la transition. La transformation des économies planifiées en économies de marché. Paris, Éditions Bréal, Licence-Master Économie, 494 p.

Badouin R. (1971). Économie rurale. Paris, Éd. A. Colin, collection U, 598 p.

Bazin G. (2007). Politique agricole commune à l'Est. Premiers résultats. Paris, La documentation française, octobre, Le courrier des pays de l'Est, $\mathrm{n}^{\circ} 1063$, p. 11-21.

Blaha J., Borzeda A. (1999). L'industrie des boissons alcoolisées en Europe centrale et orientale. Paris, La documentation française, juillet, Le courrier des pays de l'Est, $\mathrm{n}^{\circ} 441$, p. 26-46.

Commission européenne. (1998). Country report on Romania. Bruxelles, General Directorate for Agriculture, July.

Commission européenne. (2002). L'élargissement et l'agriculture : l'intégration réussie des nouveaux États membres dans la PAC. Bruxelles, SEC(2002)95 final.

Darrot C., Mouchet C. (2005). La paysannerie polonaise peut-elle être moderne et durable ? Écologie et politique, $\mathrm{n}^{\circ} 31$, p. 75-89.
Davis J.-R., Gaburici A. (1998). The economic activity of private farms in Romania during transition: just how competitive are they? Edinburgh, CERT, $48 \mathrm{p}$.

Dufumier M., Bergeret P. (2002). Analyser la diversité des exploitations agricoles. Paris, CIRAD — GRET, ministère des Affaires étrangères, Mémento de l'agronome, p. 321-344.

Duma V., Molnar M., Panduru F., Verger D. (2005). Roumanie : une agriculture de survie, après l'industrialisation forcée. Paris, INSEE, décembre, Économie et statistique, $\mathrm{n}^{\circ}$ 383-384-385, p. 193-217.

Ghib M.-L. (2009). Retraite et agriculture en Roumanie. Une indemnité viagère aux objectifs ambigus. Paris, SFER, mai-juin, Économie Rurale, $\mathrm{n}^{\circ} 311$, p. 35-48.

Ghib M.-L., Berriet-Solliec M. (2008). Mise en œuvre de la politique des structures roumaines : une nécessaire définition de l'activité agricole pour un ciblage des mesures. In « Journées INRA-SFER ». Lille, $27 \mathrm{p}$.

Holcblat N. (1994). La privatisation en Europe de l'Est : problèmes, méthodes et réalités. Paris, INSEE, Économie et statistique, $\mathrm{n}^{\circ} 279-280$, p. 101-120.

Kostov P., Lingard L. (2002). Subsistence farming in transitional economies: lessons from Bulgaria. Elsevier, Journal of Rural Studies, $\mathrm{n}^{\circ} 18$, p. 83-94.

Kostov P., Lingard L. (2004). Subsistence Agriculture in Transition Economies: its Roles and Determinants. Wiley-Blackwell, Agricultural Economics, ${ }^{\circ} 55$, p. 565-579.

Labonne M. (1985). Sur le concept de filière en économie agroalimentaire. INRA.

Lhomel E. (2007). Roumanie 2006-2007. Bourbier politique et envolée économique. Paris, La documentation française, avril, Le courrier des pays de l'Est, $\mathrm{n}^{\circ} 1062$, p. 184-196. 
Mathijs E., Noev N. (2004). Subsistence Farming in Central and Eastern Europe. Empirical Evidence from Albania, Bulgaria, Hungary, and Romania. NewYork, M.-E. Sharpe, NovemberDecember, Eastern European Economics, $\mathrm{n}^{\circ} 42$, p. 72-89.

Milhau J., Montagne R. (1968). Économie rurale. Paris, PUF, $456 \mathrm{p}$.

Moati P. (1995-1999). Méthode d'étude sectorielle. Cahiers de recherche $d u$ Crédoc, $\mathrm{n}^{\circ}$ 70, 93, 109, 118, 135.

Montaigne E. (1997). Crise biologique, différentiation des savoirs et mutation des systèmes technique et de production viticoles. Paris, C.R.P.E.E., Revue d'Économie Méridionale, $\mathrm{n}^{\circ}$ 180, p. 417-438.

Montaigne E., Couderc J.-P., Hannin H., d'Hauteville F. (2005, 2006, 2008). Bacchus - Enjeux, stratégies et pratiques dans la filière vitivinicole. Paris, Dunod, env. $300 \mathrm{p}$.

Noev N., Swinnen J.-J. (2004). The Transition of the Wine Industry, Policy and Trade in Eastern Europe and the former Soviet Union. In Anderson K., (ed.) "The World's wine Markets globalization at work", London, Edward Elgar, 368 p.

Noev N. (2007). Land, Wine, and Trade. The Transition of the Romanian Wine Sector. M.-E. Sharpe Inc., Eastern European Economics, 45(3), mai-juin, p. 76114.

Pouliquen A. (2001a). Compétitivité et revenus agricoles dans les secteurs agroalimentaires des PECO — Implications avant et après adhésion pour les marchés et les politiques de l'UE. Bruxelles, Commission européenne, $96 \mathrm{p}$.

Pouliquen A. (2001b). L'agriculture néopaysanne roumaine : le tampon social contre la relance globale. A. Colin, Revue d'études comparatives Est-Ouest, $\mathrm{n}^{\circ} 32$, p. 121-153.

Râmniceanu I. (2004). Probleme structu- rale ale agriculturii românesti în perspectiva aderarii la Uniunea Europeana (problèmes d'origine structurelle de l'agriculture roumaine dans la perspective de l'adhésion à l'Union européenne). Bucuresti, Cerope Studiul, $\mathrm{n}^{\circ} 6$.

Rizov M., Gavrilescu D., Gow H., Mathijs E. (2001). Transition and Enterprise Restructuring: The Development of Individual Farming in Romania. Elsevier, World development, $\mathrm{n}^{\circ} 29$, p. 1257 1274.

Roger A. (2008). Subversions locales et usages partisans des politiques européennes. L'exemple de la petite viticulture en Roumanie. A. Colin, Politix. Revue des sciences sociales $d u$ politique, $\mathrm{n}^{\circ} 21$, p. 179-202.

Villemin V. (1996). La Roumanie en transition. Des systèmes de production agricoles issus de la réforme agraire ou redistribution foncière : caractérisation et conditions d'émergence. Montpellier, Mémoire de fin de cycle d'ingénieur ENSAIA, CNEARC, 66 p.

Villemin V. (1997). La dynamique de la filière vitivinicole dans le département de Buzau, Roumanie. Montpellier, Diplôme d'étude approfondie, École Nationale Supérieure d'Agronomie, 78 p.

Von Hirschausen B. (2008). Les sociétés rurales roumaines face à l'irruption des programmes de développement. A. Colin, Revue d'études comparatives Est-Ouest, $\mathrm{n}^{\circ} 39$.

Von Hirschhausen B., Guest M. (2008). Intégrer les campagnes bulgares et roumaines : le défi de leurs différences. Belin, Paris, L'espace géographique, $\mathrm{n}^{\circ} 298$, p. 297-312.

Zarnoveanu B., Stefan I. (2002). Romanian Wine Industry Survey 2002. Bucarest, FAO, 40 p. 